\title{
Loss of ARID1A expression is related to shorter progression-free survival and chemoresistance in ovarian clear cell carcinoma
}

Atsuko Katagiri ${ }^{1}$, Kentaro Nakayama ${ }^{1}$, Mohammed Tanjimur Rahman ${ }^{1}$, Munmun Rahman ${ }^{1}$, Hiroshi Katagiri ${ }^{1}$, Naomi Nakayama ${ }^{2}$, Masako Ishikawa ${ }^{1}$, Tomoka Ishibashi ${ }^{1}$, Kouji Iida ${ }^{1}$, Hiroshi Kobayashi ${ }^{3}$, Yoshiro Otsuki ${ }^{3}$, Satoru Nakayama ${ }^{4}$ and Kohji Miyazaki ${ }^{1}$

${ }^{1}$ Department of Obstetrics and Gynecology, Shimane University School of Medicine, Izumo, Japan;

${ }^{2}$ Department of Biochemistry, Shimane University School of Medicine, Izumo, Japan; ${ }^{3}$ Department of

Pathology, Seirei Hamamatsu General Hospital, Hamamatsu, Japan and ${ }^{4}$ Department of Obstetrics and

Gynecology, Seirei Hamamatsu General Hospital, Hamamatsu, Japan

Recently, the ARID1A gene has been identified as a novel tumor suppressor in ovarian clear cell carcinoma. The prognostic significance of the loss of ARID1A expression is not known. The current study was designed to evaluate whether ARID1A was a prognostic factor for progression, survival, and chemoresistance in ovarian clear cell carcinoma. A total of 60 patients, who were surgically treated for primary ovarian clear cell adenocarcinoma, were enrolled. Surgical specimens were examined for ARID1A protein expression by immunohistochemistry. The correlations between the loss of ARID1A expression and clinicopathological characteristics, prognosis, and chemosensitivity were investigated. Loss of ARID1A expression was identified in $9(15.0 \%)$ of 60 ovarian clear cell carcinoma samples. Loss of ARID1A staining intensity (0+) was more frequently found in cells of clear cell carcinomas than in high-grade serous carcinomas $(P<0.01)$. Loss of ARID1A expression was significantly correlated with advanced FIGO stage and high CA125 levels $(P=0.02$, 0.01). There were no significant correlations between loss of ARID1A expression and patient age, status of residual tumor, Ki-67 labeling index, or the status of endometriosis. Loss of ARID1A correlated with shorter progression-free survival of patients with clear cell carcinomas treated with platinum-based chemotherapy $(P<0.01)$. Loss of ARID1A expression tended to correlate with shorter overall survival in patients with ovarian clear cell carcinomas treated with platinum-based chemotherapy. When data were stratified for the multivariate analysis, only the loss of ARID1A expression remained a significant $(P=0.03)$ predictor of reduced progressionfree survival. Of the 60 patients with ovarian clear cell carcinomas, 14 patients had measurable residual tumor after primary cytoreductive surgery. Tumors with loss of ARID1A expression were more likely to be chemoresistant than tumors with positive ARID1A expression $(100.0$ vs $40.0 \%, P=0.04)$. This study demonstrates that loss of ARID1A in ovarian clear cell carcinoma is a negative prognostic factor in patients treated with platinum-based chemotherapy. Measurement of ARID1A expression may be a method to predict resistance to platinum-based chemotherapy in patients with ovarian clear cell carcinoma.

Modern Pathology (2012) 25, 282-288; doi:10.1038/modpathol.2011.161; published online 18 November 2011

Keywords: ARID1A; chemoresistance; ovarian clear cell carcinoma; survival

Ovarian cancer is the most lethal gynecological malignancy in the world ${ }^{1}$ and its incidence has

Correspondence: Dr K Nakayama, MD, PhD, Department of Obstetrics and Gynecology, Shimane University School of Medicine, 89-1Enyacho, Izumo, Shimane 693-8501, Japan.

E-mail: kn88@med.shimane-u.ac.jp

Received 31 May 2011; revised 5 September 2011; accepted 6 September 2011; published online 18 November 2011 increased in the last decade. Despite a dramatic improvement in the survival rate of patients following the introduction of platinum-taxane combination chemotherapy, ${ }^{2-4}$ the 5-year overall survival rate remains around $45 \%$. Ovarian carcinoma is subdivided into four major histological types: serous, mucinous, endometrioid, and clear cell. Of these, ovarian clear cell carcinoma, which constitutes around $25 \%$ of cases in Japan, is genetically 
distinct from the other histological types. Ovarian clear cell carcinoma is diagnosed at an early stage in $\sim 60 \%$ of patients; however, it carries a worse prognosis because resistance to conventional cytotoxic chemotherapeutic agents is common even with early disease. ${ }^{5-7}$ Identification of the molecular pathways, which confer chemoresistance in ovarian clear cell carcinoma is essential for the development of novel targeted therapeutic agents.

Unlike serous carcinomas, morphological and molecular studies have clearly linked clear cell carcinoma to an endometrosis precursor lesion, which, in a stepwise fashion, develops atypia before progressing to invasive carcinoma. ${ }^{8-12}$ Mutations in PIK3CA ${ }^{13}$ and genomic amplification of chr20q13.2 ${ }^{14}$ are presently the most common molecular genetic alterations in ovarian clear cell carcinoma. Our recently published genome-wide sequencing analysis of ovarian clear cell carcinomas, however, has led to the discovery of somatic mutations in the novel tumor suppressor ARID1A. ${ }^{15}$

BAF250a, the protein encoded by ARID1A (the AT-rich interactive domain 1A (SWI-like) gene), ${ }^{16,17}$ is one of the accessory subunits of the SWI-SNF complex. The SWI-SNF chromatin remodeling complex, present in all eukaryotes, regulates gene expression for many cellular processes, including development, differentiation, proliferation, DNA repair, and tumor suppression. ${ }^{18}$ The complex uses ATP to mobilize nucleosomes, thereby modulating the accessibility of promoters to transcriptional activation or repression. ARID1A rearrangement has been found in a breast cancer cell line, and an ARID1A deletion has been identified in a lung cancer cell line. These suggest that ARID1A is a tumor-suppressor gene. ${ }^{19}$ The role of ARID1A as a tumor suppressor is also supported by the nature and pattern of its mutations in ovarian clear cell carcinoma. ${ }^{15,20}$

Although ARID1A mutation is proving to be a frequent alteration in ovarian clear cell carcinoma, its clinicopathological and prognostic role is unclear. The current study examined the prognostic significance of loss of ARID1A expression in ovarian clear cell carcinoma by investigating the relationship between ARID1A immunohistochemical expression and various clinicopathological variables in ovarian clear cell carcinoma. Finally, we assessed the utility of ARID1A as a prognostic and chemoresistance marker in ovarian clear cell carcinoma.

\section{Materials and methods}

\section{Tissue Samples}

Formalin-fixed, paraffin-embedded tissue samples of 77 ovarian cancers, including 60 clear cell carcinomas, and 17 high-grade serous adenocarcinomas were used in this study. Samples were obtained from the Department of Obstetrics and Gynecology at the Shimane University Hospital and the Department of Obstetrics and Gynecology at Seirei Hamamatsu General Hospital. Diagnosis was based on the conventional morphological examination of sections stained with hematoxylin and eosin (H\&E), and tumors were classified according to the WHO classification. Tumor staging was performed according to the International Federation of Gynecology and Obstetrics (FIGO) classification. All patients were primarily treated with cytoreductive surgery and adjuvant platinum and taxane or CPT-11 chemotherapy (CBDCA AUC 5 with paclitaxel $175 \mathrm{mg} / \mathrm{m}^{2}$ or docetaxel $70 \mathrm{mg} / \mathrm{m}^{2}$ or CDDP $60 \mathrm{mg} / \mathrm{m}^{2}$ with CPT-11 $180 \mathrm{mg} / \mathrm{m}^{2}$ ). All patients received 6-12 cycles of this combination regimen. Adjuvant chemotherapy was administered to all patients. The treatment included the following: $46.6 \%$ received (28/60) paclitaxal and carboplatin combination, $35 \%(21 / 60)$ CPT-11 and cisplatin combination, $18.4 \%(11 / 60)$ docetaxel and carboplatin combination. Acquisition of tissue specimens and clinical information was approved by an institutional review board (Shimane University and Seirei Hamamatsu General Hospital). The paraffin tissue blocks were organized into tissue microarrays, each made by removing 3-mm diameter cores of tumor from the block. Selection of the area to core was made by a gynecologic oncologist (K.N.) and pathology technician (K.I.) and was based on a review of the H\&E slides.

\section{Immunohistochemistry}

Expression levels of ARID1A and Ki-67 were assessed by immunohistochemistry. The antibodies used in this study were a mouse monoclonal antibody to ARID1A (BAF250a) (Santa Cruz Biotechnology, Santa Cruz, CA) and the mouse monoclonal antibody to Ki-67, MIB1 (DAKO, Carpinteria, CA, USA). Immunohistochemistry studies for ARID1A and Ki-67 were performed on tissue microarrays at a dilution of 1:50 or 1:100 followed by detection with the En Vision + System using the peroxidase method (DAKO). After antigen retrieval in a sodium citrate buffer, slides were incubated with antibody overnight at $4^{\circ} \mathrm{C}$. Slides for all samples were evaluated with a light microscope by two researchers who were blind to the clinicopathologic factors. ARID1A immunoreactivity was scored by the two investigators as follows: 0 : undetectable, $1+$ : weakly positive, $2+$ : moderately positive, and $3+$ : intensely positive. The evaluation criteria for Ki-67 has been detailed in a previous report. ${ }^{21}$

\section{Statistical Methods for Clinical Correlation}

Overall survival was calculated from the date of diagnosis to the date of death or last follow-up. Survival data were plotted as Kaplan-Meier curves, and the statistical significance was determined by 
the Log-rank test. A multivariate prognostic analysis was performed using a Cox proportional hazards model. Data were censored when patients were lost to follow-up. The $\chi^{2}$-test or Fisher's exact test was used for comparisons of categorical data.

\section{Results}

\section{Identification of Loss of ARID1A Expression in Ovarian Clear Cell Carcinomas}

Loss of ARID1A expression was identified in 9 $(15.0 \%)$ of 60 ovarian clear cell carcinoma samples. We also analyzed ARID1A expression in 17 highgrade serous adenocarcinoma samples to confirm whether loss of ARID1A is ovarian clear cell carcinoma specific. Expectedly, none of the highgrade ovarian serous adenocarcinoma samples $(0 / 17$, $0 \%$ ) showed loss of ARID1A expression. Immunoreactivity of ARID1A was detected in tumor cell nuclei (Figure 1). Loss of ARID1A staining intensity $(0+)$ was more frequently found in cells of ovarian clear cell carcinomas than in high-grade ovarian serous adenocarcinomas $\left(P<0.01, \chi^{2}\right.$-test) (Table 1$)$.

\section{Relationship Between the Loss of ARID1A Expression and Clinicopathologic Factors}

Results in Table 2 demonstrate that loss of ARID1A expression significantly correlated with advanced FIGO stage and high CA125 levels $(P=0.02,0.01)$. There were no significant correlations between loss of ARID1A expression and patient age, status of residual tumor, Ki-67 labeling index or the status of endometriosis.

Patients were stratified into one of two groups depending on ARID1A immunohistochemical intensity. Loss of ARID1A (ARID1A immunointensity 0 +) was observed in $15 \%(9 / 60)$ of the analyzed tumors.

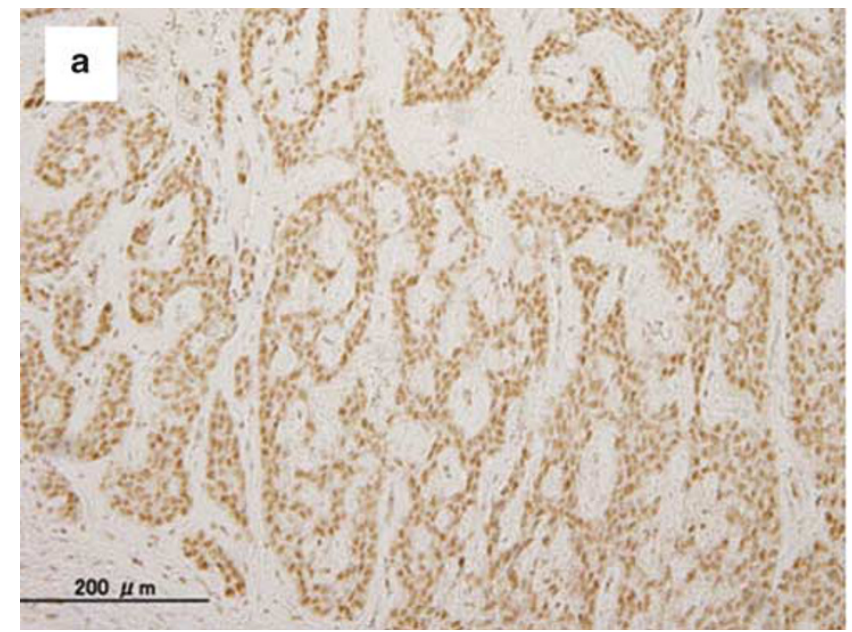

\section{Effect of Loss of ARID1A Protein Expression on Progression-Free Survival}

We looked for a possible prognostic effect of loss of ARID1A protein expression on progression-free survival. Kaplan-Meier estimates of progressionfree/overall survival are plotted in Figure 2. Of the total of 60 patients diagnosed at stages I-IV, nine patients with loss of ARID1A expression had a shorter progression-free survival than those with positive ARID1A expression $(P<0.01$; Log-rank test) (Figure 2a). A univariate analysis demonstrated that FIGO stage III, IV $(P<0.01$; Log-rank test $)$, CA125 levels $(P=0.01$; Log-rank test $)$, residual tumor $\geqq 2 \mathrm{~cm}(P<0.01$; Log-rank test), and loss of ARID1A expression $(P<0.01$; Log-rank test $)$ correlated with shorter progression-free survival. When the data were stratified in the multivariate analysis, only loss of ARID1A expression remained significant $(P=0.03)$ for shorter disease-free survival (Table 3$)$.

\section{Effect of Loss of ARID1A Protein Expression on Overall Survival}

Next, we examined any overall prognostic effect of loss of ARID1A protein expression. Loss of ARID1A expression tended to correlate with shorter overall survival in patients with ovarian clear cell carcinomas treated with platinum-based chemotherapy $(P=0.15$; Log-rank test) (Figure $2 b)$. The univariate

Table 1 The relationship between ARID1A expression and histological subtype

\begin{tabular}{lccc}
\hline & Negative (\%) & Positive (\%) & P-value \\
\hline OCCCs & $9(15)$ & $51(85)$ & $P<0.01$ \\
HSOCs & $0(0)$ & $17(100)$ & \\
\hline
\end{tabular}

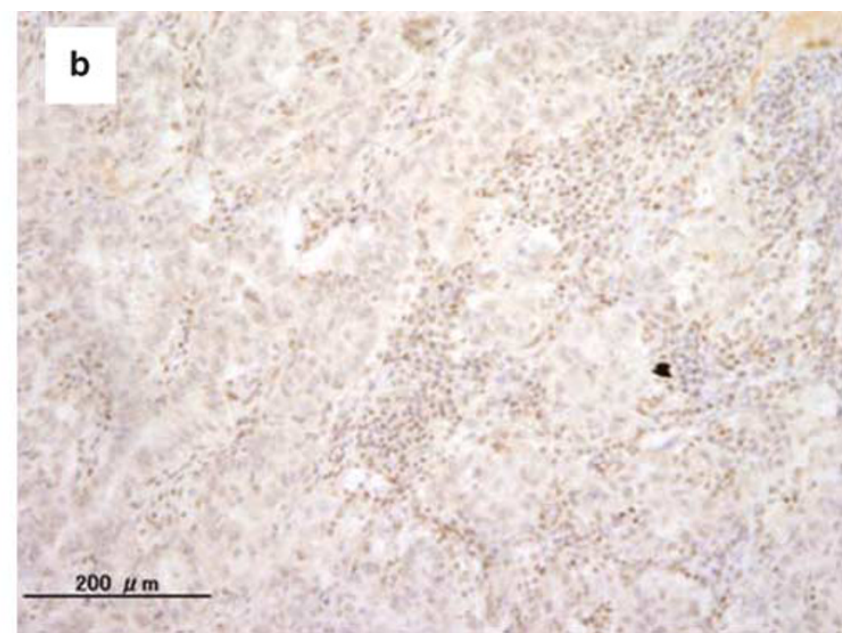

Figure 1 (a) Immunoreactivity of ARID1A in ovarian clear cell carcinoma tissue. Intense immunoreactivity is present in the nuclei of ovarian clear cell carcinoma cells (upper left panel). (b) A case with negative staining of ARID1A (upper right panel). Stromal cells showed positive staining ARID1A. 
Table 2 Association between ARID1A expression and clinicopathological factors in patients with ovarian clear cell carcinoma

\begin{tabular}{|c|c|c|c|c|}
\hline \multirow[t]{2}{*}{ Factors } & \multirow[t]{2}{*}{ Patients } & \multicolumn{2}{|c|}{ ARID1A immunostaining } & \multirow[t]{2}{*}{$\mathrm{P}$-value } \\
\hline & & Negative & Positive & \\
\hline \multicolumn{5}{|l|}{ FIGO stage } \\
\hline I, II & 45 & 4 & 41 & 0.02 \\
\hline III, IV & 15 & 5 & 10 & \\
\hline \multicolumn{5}{|l|}{ CA125, $\mathrm{U} / \mathrm{ml}$} \\
\hline$<90$ & 30 & 1 & 29 & 0.01 \\
\hline$\geqq 90$ & 30 & 8 & 22 & \\
\hline \multicolumn{5}{|l|}{ Age (years) } \\
\hline$<54$ & 30 & 5 & 25 & 0.72 \\
\hline$\geqq 54$ & 30 & 4 & 26 & \\
\hline \multicolumn{5}{|l|}{ Endometriosis } \\
\hline Without & 32 & 5 & 27 & 0.89 \\
\hline With & 28 & 4 & 24 & \\
\hline \multicolumn{5}{|l|}{ Ki-67 } \\
\hline Low & 30 & 4 & 26 & 0.72 \\
\hline High & 30 & 5 & 25 & \\
\hline \multicolumn{5}{|l|}{ Chemotherapy } \\
\hline CPT-11+CDDP & 21 & 6 & 15 & 0.03 \\
\hline CBDCA+taxane & 39 & 3 & 36 & \\
\hline \multicolumn{5}{|l|}{ Residual tumor } \\
\hline$<2 \mathrm{~cm}$ & 48 & 6 & 42 & 0.28 \\
\hline$\geqq 2 \mathrm{~cm}$ & 12 & 3 & 9 & \\
\hline
\end{tabular}

analysis demonstrated that FIGO stage III, IV $(P<0.01$; Log-rank test $)$ CA125 levels $(P=0.01$; Log-rank test), and residual tumor $\geqq 2 \mathrm{~cm}(P<0.01$; Log-rank test) significantly correlated with shorter overall survival. When data were stratified for the multivariate analysis, only residual tumor $(\geqq 2 \mathrm{~cm})$ remained a significant $(P=0.04$, respectively) predictor for shorter overall survival (Table 4).

\section{Relationship Between Loss of ARID1A Expression and Chemotherapeutic Response}

Of the 60 ovarian clear cell carcinoma patients, 14 patients had measurable residual disease after the primary cytoreductive surgery. Of these 14 patients, six $(42.8 \%)$ responded to chemotherapy and eight $(57.2 \%)$ did not. Tumors with loss of ARID1A were more likely to be resistant to primary adjuvant chemotherapy than were tumors with positive ARID1A expression (100.0 vs $40.0 \%, P=0.04$ ) (Table 5).

\section{Discussion}

ARID1A, a recently identified tumor suppressor gene, is mutated in $\sim 50 \%$ of ovarian clear cell and $30 \%$ of ovarian endometrioid carcinomas. ${ }^{15,20}$ The clinical significance of ARID1A mutation is unknown. Recently, Maeda et $a l^{22}$ reported that
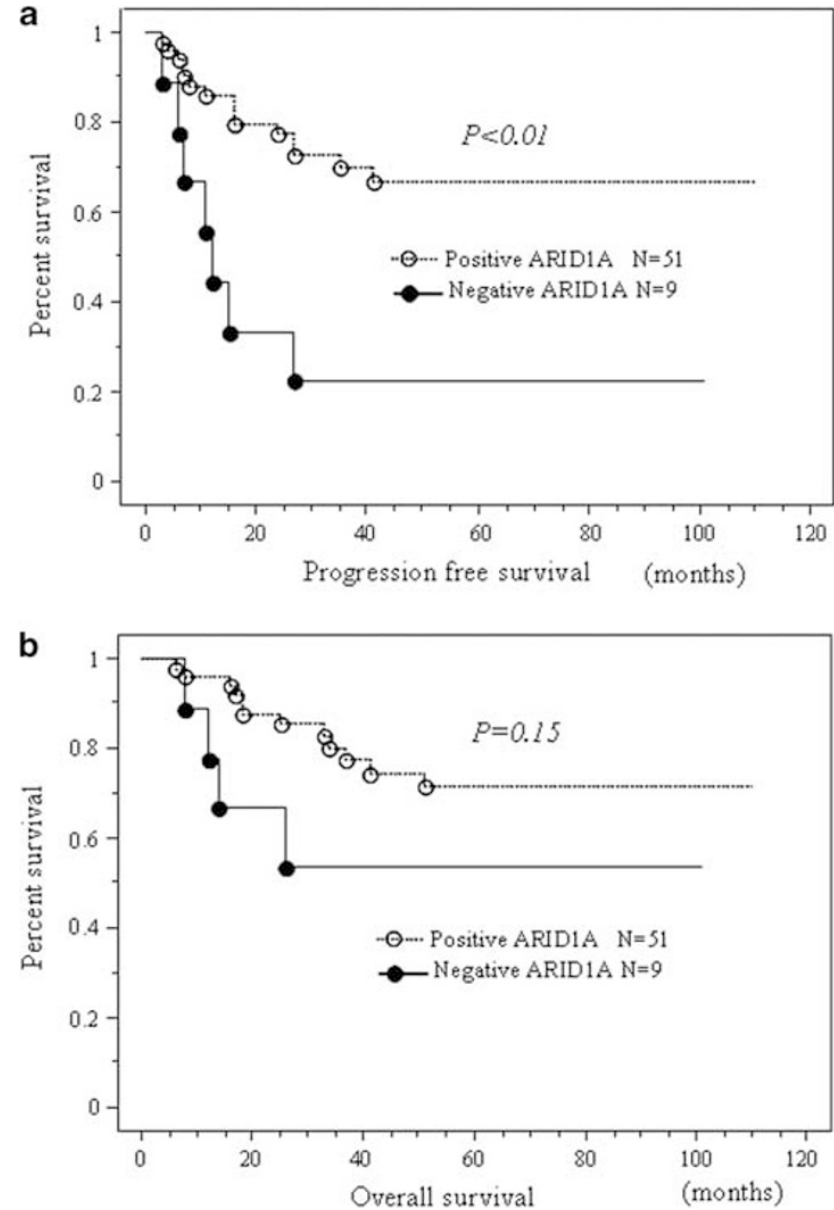

Figure 2 Loss of ARID1A expression correlates with shorter progression-free survival in ovarian clear cell carcinoma patients who received primary cytoreductive surgery followed by standard platinum-based chemotherapy. (a) Kaplan-Meier survival analysis showing that negative ARID1A expression (solid line, $n=9$ ) is associated with a shorter progression-free survival than positive ARID1A expression (dashed line, $n=51)(P<0.01$, Log-rank test). (b) Negative ARID1A expression (solid line, $n=9$ ) had a statistically nonsignificant effect on overall survival in ovarian clear cell carcinoma patients who received primary cytoreductive surgery followed by standard platinum-based chemotherapy $(P=0.15$, Log-rank test $)$.

ARID1A mutation was significantly correlated with the loss of ARID1A protein expression using immunohistochemistry. Therfore, we evaluated the clinicopathological and prognostic significance of ARID1A immunoreactivity in ovarian clear cell carcinoma.

In the current study, the higher frequency of loss of ARID1A expression in ovarian clear cell carcinomas compared with ovarian high-grade serous adenocarcinomas is a finding of interest. It supports the hypothesis that ovarian clear cell carcinomas may be distinguished from their more common serous counterparts based on characteristic alterations in gene sequences and expression. ${ }^{12,23}$ Additionally, this observation further supports the theory that each histological type of ovarian carcinoma arises from a distinct molecular pathway. ${ }^{24,25}$ 
Table 3 Univariate and multivariate analysis of progression-free prognostic factors in patients with ovarian clear cell carcinoma

\begin{tabular}{|c|c|c|c|c|c|c|c|}
\hline \multirow[t]{2}{*}{ Factors } & \multirow[t]{2}{*}{ Patients } & \multicolumn{3}{|c|}{ Univariate } & \multicolumn{3}{|c|}{ Multivariate } \\
\hline & & Hazard ratio & $95 \% C I$ & $\mathrm{P}$-value & Hazard ratio & $95 \% C I$ & $\mathrm{P}$-value \\
\hline \multicolumn{8}{|l|}{ FIGO stage } \\
\hline I, II & 45 & & & & & & \\
\hline III, IV & 15 & 9.2 & $3.2-26.8$ & $<0.01$ & 2.3 & $0.6-8.9$ & 0.22 \\
\hline \multicolumn{8}{|l|}{ CA125, U/ml } \\
\hline$<90$ & 30 & & & & & & \\
\hline$\geqq 90$ & 30 & 5.4 & $1.5-18.8$ & 0.01 & 1.6 & $0.5-4.9$ & 0.41 \\
\hline \multicolumn{8}{|l|}{ Age (years) } \\
\hline$<54$ & 30 & & & & & & \\
\hline$\geqq 54$ & 30 & 2.1 & $0.7-6.0$ & 0.17 & NA & NA & NA \\
\hline \multicolumn{8}{|l|}{ Endometriosis } \\
\hline Without & 32 & & & & & & \\
\hline With & 28 & 1.8 & $0.7-4.5$ & 0.19 & NA & NA & NA \\
\hline \multicolumn{8}{|l|}{ Ki-67 } \\
\hline Low & 30 & & & & & & \\
\hline High & 30 & 1.7 & $0.7-4.0$ & 0.23 & NA & NA & NA \\
\hline \multicolumn{8}{|l|}{ Residual tumor } \\
\hline$<2 \mathrm{~cm}$ & 48 & & & & & & \\
\hline$\geqq 2 \mathrm{~cm}$ & 12 & 6.1 & $2.6-14.2$ & $<0.01$ & 3.1 & $0.9-10.9$ & 0.07 \\
\hline \multicolumn{8}{|l|}{ Chemotherapy } \\
\hline CPT-11+CDDP & 21 & & & & & & \\
\hline CBDCA+taxane & 39 & 0.6 & $0.3-1.4$ & 0.62 & NA & NA & NA \\
\hline \multicolumn{8}{|c|}{ ARID1A immunostaining } \\
\hline Negative & 9 & & & & & & \\
\hline Positive & 51 & 4 & $1.6-9.9$ & $<0.01$ & 3.1 & $1.1-8.6$ & 0.03 \\
\hline
\end{tabular}

Our most notable finding is that loss of ARID1A in ovarian clear cell carcinomas predicted a shorter progression-free interval. To date, there are a few molecular markers that predict the risk of early tumor recurrence in ovarian clear cell carcinomas. ${ }^{26,27}$ Therefore, loss of ARID1A expression may have the potential to be used alone or in combination with other markers to identify ovarian clear cell carcinoma patients who are more susceptible to early recurrence. This is important because at least $60 \%$ of advanced-stage ovarian clear cell carcinoma patients with a complete response to primary therapy ultimately develop recurrent disease. ${ }^{28}$ Taken together, these observations may have an impact on clinical management. Patients with recurrent ovarian clear cell carcinomas derive the most benefit from secondary cytoreduction if recurrent tumors are small and localized. ${ }^{28-32}$ Therefore, patients with loss of ARID1A may be followed more frequently to detect recurrences early enough to benefit from either secondary cytoreductive surgery or secondline chemotherapy. A recent study has shown that a significant correlation exists between the loss of ARID1A immunostaining and ARID1A mutation. ${ }^{20}$ This indicates that the immunohistochemical analysis of ARID1A may be a useful strategy for mutational analysis in the clinical setting.
In the current study, the percentage of ovarian clear cell carcinomas with negative ARID1A is lower than what has been previously published. ${ }^{20,22}$ Lack of a consistent the percentage may be the result of study biases. First, differences in the methodological protocols, including the source and dilution of the antibodies and the systems used to assess negative immunohistochemical reactions, may produce significant variability among results. Second, the sample sizes of these studies, including the present one, are relatively small. Larger studies are required to definitively establish the percentage of loss of ARID1A expression on ovarian clear cell carcinomas.

In the current study, patients with ARID1Anegative tumors had a significantly inferior response to chemotherapy when compared with patients who had ARID1A tumors. The fact that loss of ARID1A expression assessed by immunohistochemistry is an independent predictor of the progression-free interval in ovarian clear cell carcinomas attests to its value as a marker for predicting platinum resistance. In general, the prognosis of patients with clear cell adenocarcinoma is poor, which is largely due to a low response rate to conventional platinum- or taxane-based chemotherapy. ${ }^{5,6,33}$ In a large retrospective study comparing 101 cases of clear cell carcinoma and 235 cases of serous 
Table 4 Univariate and multivariate analysis of overall prognostic factors in patients with ovarian clear cell carcinoma

\begin{tabular}{|c|c|c|c|c|c|c|c|}
\hline \multirow[t]{2}{*}{ Factors } & \multirow[t]{2}{*}{ Patients } & \multicolumn{3}{|c|}{ Univariate } & \multicolumn{3}{|c|}{ Multivariate } \\
\hline & & Hazard ratio & $95 \% C I$ & $\mathrm{P}$-value & Hazard ratio & $95 \% C I$ & $\mathrm{P}$-value \\
\hline \multicolumn{8}{|l|}{ FIGO stage } \\
\hline I, II & 45 & & & & & & \\
\hline III, IV & 15 & 6.3 & $2.7-14.7$ & $<0.01$ & 1.8 & $0.3-10.6$ & 0.5 \\
\hline \multicolumn{8}{|l|}{ CA125, U/ml } \\
\hline$<90$ & 30 & & & & & & \\
\hline$\geqq 90$ & 30 & 3.5 & $1.4-9.0$ & 0.01 & 3.2 & $0.8-13.0$ & 0.1 \\
\hline \multicolumn{8}{|l|}{ Age (years) } \\
\hline$<54$ & 30 & & & & & & \\
\hline$\geqq 54$ & 30 & 1.7 & $0.7-4.1$ & 0.23 & NA & NA & NA \\
\hline \multicolumn{8}{|l|}{ Endometriosis } \\
\hline Without & 32 & & & & & & \\
\hline With & 28 & 1.8 & $0.7-4.5$ & 0.1 & NA & NA & NA \\
\hline \multicolumn{8}{|l|}{ Ki-67 } \\
\hline Low & 30 & & & & & & \\
\hline High & 30 & 2.6 & $0.9-7.5$ & 0.08 & NA & NA & NA \\
\hline \multicolumn{8}{|l|}{ Residual tumor } \\
\hline$<2 \mathrm{~cm}$ & 48 & & & & & & \\
\hline$\geqq 2 \mathrm{~cm}$ & 12 & 10 & $3.7-28.3$ & $<0.01$ & 5.5 & $1.2-25.6$ & 0.03 \\
\hline \multicolumn{8}{|l|}{ Chemotherapy } \\
\hline CPT-11+CDDP & 21 & & & & & & \\
\hline CBDCA+taxane & 39 & 1 & $0.4-2.9$ & 0.92 & NA & NA & NA \\
\hline \multicolumn{8}{|c|}{ ARID1A immunostaining } \\
\hline Negative & 9 & & & & & & \\
\hline Positive & 51 & 2.3 & $0.7-7.2$ & 0.15 & NA & NA & NA \\
\hline
\end{tabular}

Table 5 The relationship between ARID1A (BAF250a) expression and platinum-based chemotherapeutic response

\begin{tabular}{lccc}
\hline & Responder (\%) & Non-responder (\%) & $\mathrm{P}$ \\
\hline Negative & $0(0)$ & $4(100)$ & 0.0404 \\
Positive & $6(60)$ & $4(40)$ & \\
\hline
\end{tabular}

adenocarcinoma, Sugiyama et $a l^{5}$ showed that survival rates of stage III clear cell carcinoma patients were significantly lower than those of stage III serous adenocarcinoma patients. In their study, the response rate to platinum-based chemotherapy in patients with clear cell carcinoma was significantly lower than that in patients with serous adenocarcinoma. Currently, little is known about the role ARID1A has in chemoresistance. Our results suggest a need to investigate the possible link between loss of ARID1A expression and chemoresistance in ovarian clear cell carcinoma.

In the current study, all the patients were treated with either platunum and taxane combination or platinum and CPT-11 combination as the primary adjuvant regimen. Patients whose disease recurred after primary platinum-based chemotherapy were then treated with various second-line chemotherapy agents including liposomal doxorubicin, gemcitabine, and docetaxel. Differing responses to these agents may have masked the effect of loss of ARID1A expression on overall survival in patients with ovarian clear cell carcinoma. It is unclear if the effect of ARID1A expression on platinum sensitivity has a primary role in the survival patterns seen in this study. Additional prospective studies that analyze ARID1A function in patients treated with clearly defined chemotherapeutic regimens are required to answer this question.

To our knowledge, this is the first report suggesting that loss of ARID1A protein expression is a marker of poor progression-free survival and platinum resistance in ovarian clear cell carcinomas. This study is limited by its small size given the relative rarity of ovarian clear cell carcinoma. Larger prospective trials are needed to confirm our findings and to more fully explore the role of ARID1A in ovarian clear cell carcinoma behavior.

\section{Acknowledgement}

This study was supported by grants from the Ministry of Education, Culture, Sports, Science and Technology in Japan and the Kanae Research Foundation. 


\section{Disclosure/conflict of interest}

The authors declare no conflict of interest.

\section{References}

1 Wingo PA, Tong T, Bolden S. Cancer statistics 1995. CA Cancer J Clin 1995;45:8-30.

2 Bristow RE, Montz FJ, Lagasse LD, et al. Survival impact of surgical cytoreduction in stage IV epithelial ovarian cancer. Gynecol Oncol 1999;72:278-287.

3 Markman M, Liu PY, Wilczynski S, et al. Phase III randomized trial of 12 versus 3 months of maintenance paclitaxel in patients with advanced ovarian cancer after complete response to platinum and paclitaxelbased chemotherapy: a Southwest Oncology Group and Gynecologic Oncology Group trial. J Clin Oncol 2003;21:2460-2465.

4 McGuire WP, Hoskins WJ, Brady MF, et al. Cyclophosphamide and cisplatin compared with paclitaxel and cisplatin in patients with stage III and stage IV ovarian cancer. N Engl J Med 1996;334:1-6.

5 Sugiyama T, Kamura T, Kigawa J, et al. Clinical characteristics of clear cell carcinoma of the ovary: a distinct histologic type with poor prognosis and resistance to platinum-based chemotherapy. Cancer 2000;88:2584-2589.

6 Takano M, Kikuchi Y, Yaegashi N, et al. Clear cell carcinoma of the ovary: a retrospective multicentre experience of 254 patients with complete surgical staging. Br J Cancer 2006;94:1369-1374.

7 Kobayashi H, Yamada Y, Kanayama S, et al. The role of hepatocyte nuclear factor-1beta in the pathogenesis of clear cell carcinoma of the ovary. Int J Gynecol Cancer 2009;19:471-479.

8 Veras E, Mao TL, Ayhan A, et al. Cystic and adenofibromatous clear cell carcinomas of the ovary: distinctive tumors that differ in their pathogenesis and behavior: a clinicopathologic analysis of 122 cases. Am J Surg Pathol 2009;33:844-853.

9 Fukunaga M, Nomura K, Ishikawa E, et al. Ovarian atypical endometriosis: its close association with malignant epithelial tumours. Histopathology 1997;30: 249-255.

10 Erzen M, Rakar S, Klancnik B, et al. Endometriosisassociated ovarian carcinoma (EAOC): an entity distinct from other ovarian carcinomas as suggested by a nested case-control study. Gynecol Oncol 2001;83:100-108.

11 Sato N, Tsunoda H, Nishida M, et al. Loss of heterozygosity on 10q23.3 and mutation of the tumor suppressor gene PTEN in benign endometrial cyst of the ovary: possible sequence progression from benign endometrial cyst to endometrioid carcinoma and clear cell carcinoma of the ovary. Cancer Res 2000;60:7052-7056.

12 Marquez RT, Baggerly KA, Patterson AP, et al. Patterns of gene expression in different histotypes of epithelial ovarian cancer correlate with those in normal fallopian tube, endometrium, and colon. Clin Cancer Res 2005; 11:6116-6126.

13 Kuo KT, Mao TL, Jones S, et al. Frequent activating mutations of PIK3CA in ovarian clear cell carcinoma. Am J Pathol 2009;174:1597-1601.

14 Kuo KT, Mao TL, Chen X, et al. DNA copy numbers profiles in affinity-purified ovarian clear cell carcinoma. Clin Cancer Res 2010;16:1997-2008.
15 Jones S, Wang TL, Shih Ie M, et al. Frequent mutations of chromatin remodeling gene ARID1A in ovarian clear cell carcinoma. Science 2010;330:228-231.

16 Sif S, Saurin AJ, Imbalzano AN, et al. Purification and characterization of mSin3A-containing Brg1 and hBrm chromatin remodeling complexes. Genes Dev 2001;15: 603-618.

17 Wang W, Xue Y, Zhou S, et al. Diversity and specialization of mammalian SWI/SNF complexes. Genes Dev 1996;10:2117-2130.

18 Reisman D, Glaros S, Thompson EA. The SWI/SNF complex and cancer. Oncogene 2009;28:1653-1668.

19 Huang J, Zhao YL, Li Y, et al. Genomic and functional evidence for an ARID1A tumor suppressor role. Genes Chromosomes Cancer 2007;46:745-750.

20 Wiegand KC, Shah SP, Al-Agha OM, et al. ARID1A mutations in endometriosis-associated ovarian carcinomas. N Engl J Med 2010;363:1532-1543.

21 Nakayama K, Takebayashi Y, Hata K, et al. The proliferative activity of ovarian tumors of low malignant potential differs from that of ovarian carcinoma. Anticancer Res 2003;23:4657-4662.

22 Maeda D, Mao TL, Fukayama M, et al. Clinicopathological significance of loss of ARID1A immunoreactivity in ovarian clear cell carcinoma. Int J Mol Sci 2010;11:5120-5128.

23 Schwartz DR, Kardia SL, Shedden KA, et al. Gene expression in ovarian cancer reflects both morphology and biological behavior, distinguishing clear cell from other poor-prognosis ovarian carcinomas. Cancer Res 2002;62:4722-4729.

24 Shih Ie M, Kurman RJ. Ovarian tumorigenesis: a proposed model based on morphological and molecular genetic analysis. Am J Pathol 2004;164:1511-1518.

25 Shih Ie M, Kurman RJ. Molecular pathogenesis of ovarian borderline tumors: new insights and old challenges. Clin Cancer Res 2005;11:7273-7279.

26 Kobel M, Xu H, Bourne PA, et al. IGF2BP3 (IMP3) expression is a marker of unfavorable prognosis in ovarian carcinoma of clear cell subtype. Mod Pathol 2009;22:469-475.

27 Ho CM, Cheng WF, Lin MC, et al. Prognostic and predictive values of E-cadherin for patients of ovarian clear cell adenocarcinoma. Int J Gynecol Cancer 2010;20:1490-1497.

28 Diaz-Montes TP, Bristow RE. Secondary cytoreduction for patients with recurrent ovarian cancer. Curr Oncol Rep 2005;7:451-458.

29 Harter P, du Bois A. The role of surgery in ovarian cancer with special emphasis on cytoreductive surgery for recurrence. Curr Opin Oncol 2005;17:505-514.

30 Gadducci A, Iacconi P, Cosio S, et al. Complete salvage surgical cytoreduction improves further survival of patients with late recurrent ovarian cancer. Gynecol Oncol 2000;79:344-349.

31 Gadducci A, Iacconi P, Fanucchi A, et al. Surgical cytoreduction during second-look laparotomy in patients with advanced ovarian cancer. Anticancer Res 2000;20:1959-1964.

32 Zang RY, Li ZT, Tang J, et al. Secondary cytoreductive surgery for patients with relapsed epithelial ovarian carcinoma: who benefits? Cancer 2004;100:1152-1161.

33 Goff BA, Sainz de la Cuesta R, Muntz HG, et al. Clear cell carcinoma of the ovary: a distinct histologic type with poor prognosis and resistance to platinum-based chemotherapy in stage III disease. Gynecol Oncol 1996;60:412-417. 